\title{
Perspectivas para un Modelo de Desarrollo Alternativo para el Cono Sur
}

\begin{abstract}
Alexis Saludjian
Resumen: Las últimas elecciones presidenciales en Brasil y Argentina (en 2002 y 2003 respectivamente) eran presentadas como momentos políticos propicios a cambios. Los programas electorales de los candidatos que ganarían posteriormente las elecciones (sobre todo en Argentina) prometían en el discurso una ruptura a la altura de las crisis y de los problemas causados por el neoliberalismo triufante del Consenso de Washington. Por eso parece necesario hacer un balance de las acciones gobernamentales de los dos presidentes ya en pre-campaña para las proximas elecciones (2006 y 2007). Insistiremos sobre la temática de la integración regional. Despúes de la Cumbre de Mar del Plata en diciembre 2005, de la Cumbre de Córdoba en Julio 2006 y de la incorporación de Venezuela como miembro pleno del Mercosur, aparecen nuevos interrogantes sobre el tema de la integración regional y del modelo de desarrollo. ¿Cual será el equilibrio de las fuerzas entre los diversos actores económicos y politicos en la nueva configuración del Mercosur? ¿Cual es el futuro de la Creación del Banco del Sur? ¿Simple reemplazo de las institutciones internacionales (FMI, BM, BID) ya ampliamente desacreditadas por las crisis o nueva orientación de desarrollo con homogeneidad estructural y social, como prioridad política?
\end{abstract}

Palabras-clave: Integración Regional, Modelo de Desarrollo, Vulnerabilidad Interna y Externa.

Abstract: The presidential elections in Brazil and Argentina in 2002 and 2003, respectively, were presented as appropriated political moments to implement changes. The electoral programs of the candidates who would later win the elections (especially in Argentina) were promising to break the crises and problems caused by triumphant neo-liberalism of the Consensus of Washington. So, with Kirchner's period already finished and after a longer period of Lula, it seems necessary to evaluate the governmental actions of both presidents. We insist here on regional integration matters. After the Summit in Mar del Plata in December 2005, the Summit in Cordoba in July 2006 and the incorporation of Venezuela as a Mercosur's full member, the regional integration and the model of development raise new question on the topic at hand.

Which will be the balance of the forces between the diverse economic and political actors in the new configuration of the Mercosur? Which is the future of the Banco del Sur? It will just replace the international institutions (IMF, WB, IADB) already discredited by the crises or it will be a driving force for a new economic and social development, with structural and social homogeneity as its political priority?

Keywords: Regional Integration, Economic and Social Development, Mercosur.

\footnotetext{
(*) Doctor en Ciencias Económicas, CEPN-Univ. Paris 13 y SEPLA, Professor Adjunto do IE-UFRJ. A la hora de escribir este tex to (inicio de octubre del 2006) no se conocia el resultado de la segunda vuelta de la elección presidencial en Brasil entre Lula y Alckimin. La reelección de Lula en octubre del 2006 y la politica seguida desde entonces no invalida los elementos presentados en este articulo. Una versión preliminar fue preparada ser presentada para el Simposio: Integración Regional del Congreso CEISAL 2007 "Enfoque sobre el nuevo regionalismo: entre dinámica interna y proyección internacional”, Bruselas, abril 2007.E-mail: alex_saludj@yahoo.fr. Recebido em: 3.11.08 e aceito em: 2.12.08.
} 


\section{INTRODUCCIÓN}

Según algunos economistas y centros políticos de decisiones, América latina estaría en el inicio de un nuevo ciclo de crecimiento fuerte y duradero. El caso del crecimiento de la economía argentina de casi $8 \%$ desde 2003 sería el mejor ejemplo de este nuevo ciclo de crecimiento. Brasil y México - a un nível menor — crecen respectivamente 3,8\% y 3,7\% desde 2004. A la luz de la situación actual parece difícil creer que 4 ó 5 años atras toda la región sufría una crisis profunda esencialmente tras la catástrofe argentina (1999-2002), las graves crisis políticas (consécuencias de los problemas economicos) en varios paises (Bolivia, Ecuador, Venezuela) y los ataques especulativos antes de la elección de 2002 en Brasil (Gráfico 1):

\section{Gráfico 1 - Tasa de crecimiento del PIB en América Latina 1996-2005 (en \%)}

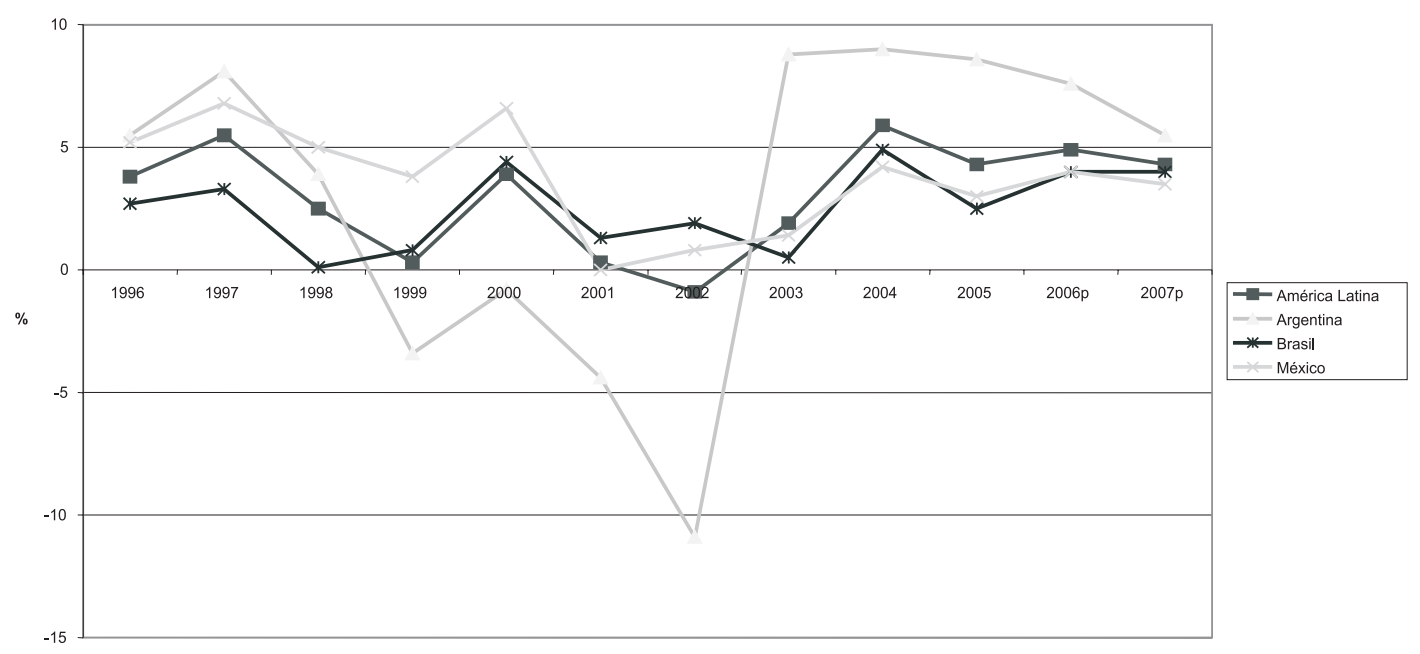

Fuente: Cepal.

En este trabajo, insistiremos sobre el nivel regional de las politicas liberales de los años 1990 a travers del concepto del Regionalismo Abierto o de Nuevo Regionalismo.

Este tipo de regionalismo tenía (y aún tiene) un objetivo doble: garantir crecimiento económico sustentable y equidad social. En las dos primeras secciones presentamos los resultados despues de más de 15 años de este tipo de integración económica. En la última sección, proponemos algunos elementos para otra integración económica, pero no solamente, en América latina ${ }^{(1)}$. En la conclusión, retomamos algunos temas actuales pendientes acerca de este proceso de regionalización en América latina.

(1) El análisis que nos lleva a estas conclusiones se encuentra de forma detallada en Saludjian, 2005-2. Presentamos aqui los resultados actualizados de este trabajo.

128 


\section{REgIONALISMO ABIERTO AL SERVICIO DE LA TRANSFORMACIÓN PRODUCTIVA CON EQUIDAD Y VOLATILIDAD MACROECONÓMICA}

El Regionalismo Abierto fue concebido como una oportunidad de crecimiento con equidad social (CEPAL, 1994) y como un medio para protegerse vis-à-vis de los otros bloques con lo que denominamos "red de seguridad regional". Hemos mostrado que los elementos teóricos utilizados para llegar a tales conclusiones (favorables a este tipo de regionalismo) así como los resultados obtenidos en términos macroeconómicos de crecimiento y de mejor inserción en la economía mundial deben ser sometidos a un examen crítico para hacer avanzar el debate teórico.

Señalamos una divergencia entre Brasil y Argentina a partir de la creación del Mercosur y el carácter inestable en el Cono Sur. En realidad, la discordancia en las trayectorias de crecimiento es más antigua y estructural. Nuestros resultados muestran dos regímenes de crecimiento diferentes en Argentina y Brasil al menos desde los años 1960 (ver Gráfico 2).

\section{Gráfico 2 - Trayectorias de crecimiento y volatilidad: Argentina, Brasil y México (1960-2005)}

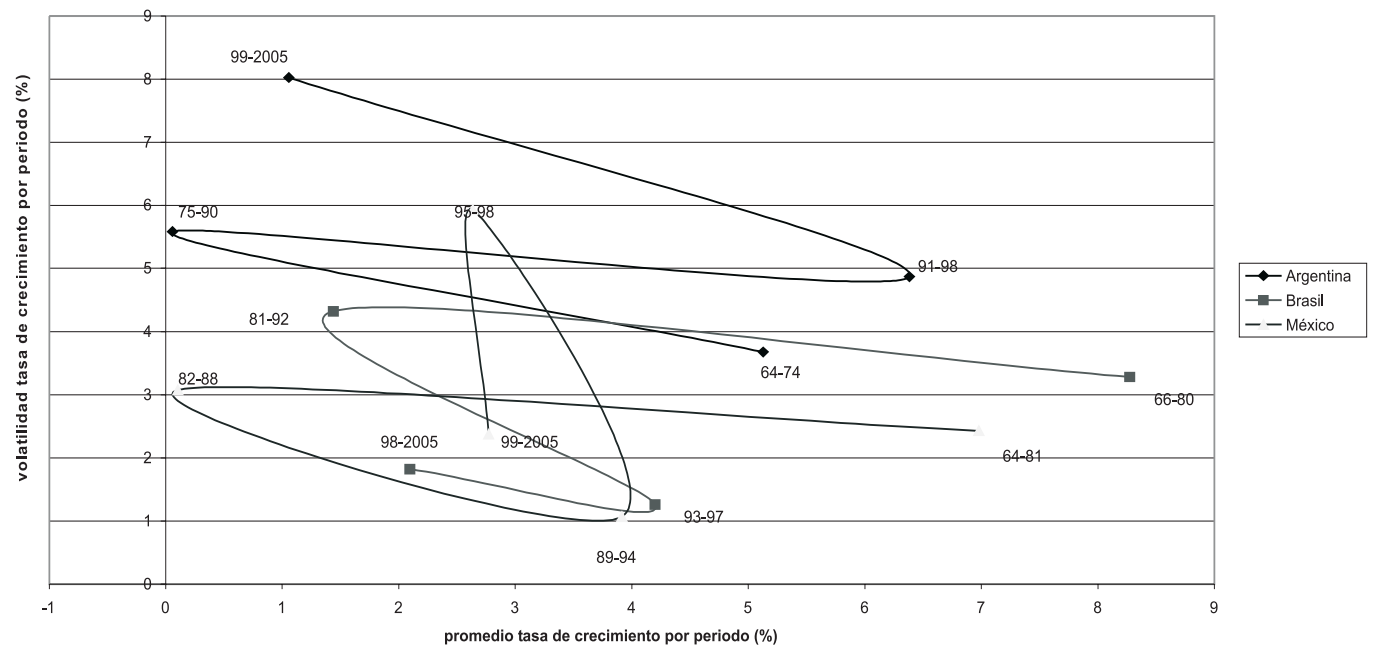

FUENTE: Elaboración del autor en base a datos WDI-BM y CEPAL.

Existen dos subgrupos en el seno del Mercosur: Brasil y Paraguay, por una parte; Argentina y Uruguay, por la otra. La ausencia de sincronización de los ciclos de crecimiento (o de recesión) constituye un factor que juega en contra de la integración regional y que puede generar efectos de contagio dentro de la zonar Gráfico 3). 


\section{Gráfico 3 - Mercosur: comercio intra-Mercosur en \% del comercio total (1980-2005)}

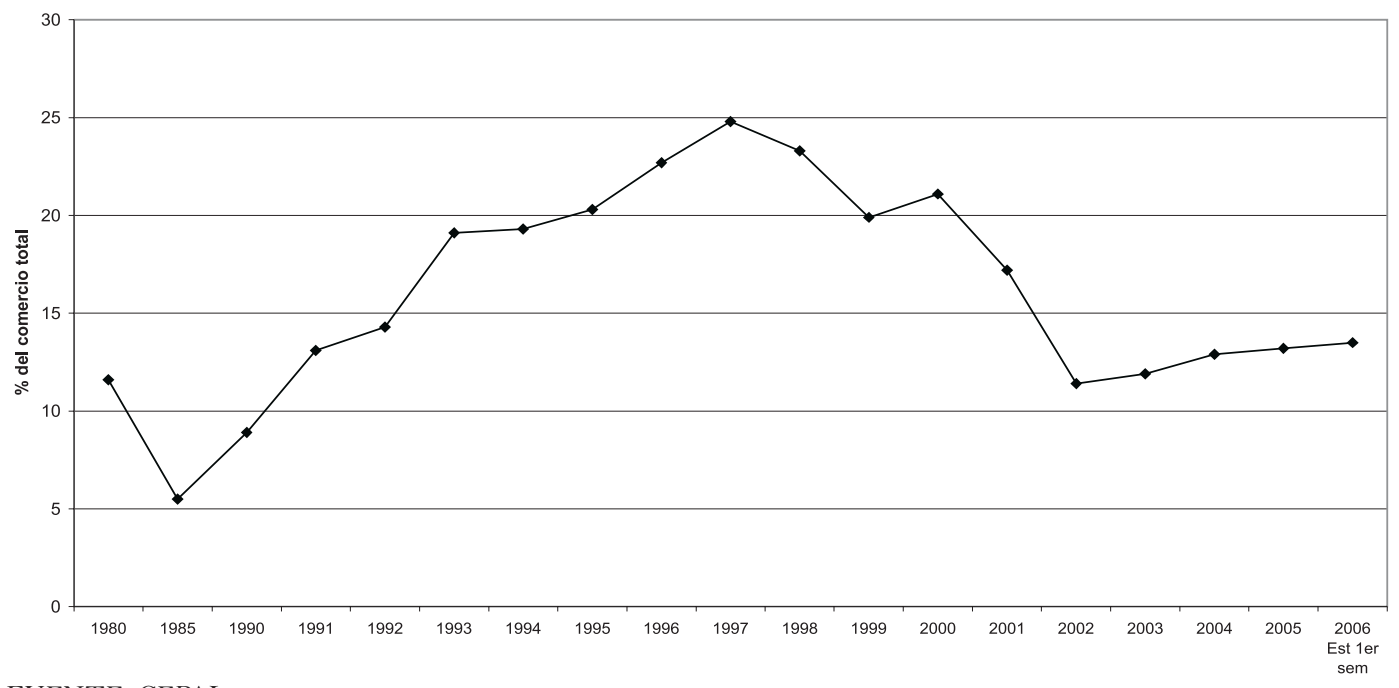

FUENTE: CEPAL

Sin embargo, estos fenómenos de contagio no prevalecieron en el Mercosur durante los años 1990. Las crisis repetidas de los países miembros tuvieron evidentemente un efecto sobre sus vecinos y asociados, pero estas crisis se deben en mayor medida a la fragilidad estructural de las economías después de las reformas liberales y la apertura comercial de los años 1980-1990. El rol del régimen de cambio fue especialmente discutido en tanto tuvo un lugar determinante durante los años 1990. Si bien la elección óptima de un régimen de cambio no es el único elemento que determina el éxito o el fracaso de la inserción de estos países en la economía mundial, hemos sugerido que la dolarización completa y el abandono de la soberanía monetaria no constituyen una solución viable para los países de la zona, a menos que se disuelva el Mercosur (y las naciones que lo componen) en el ALCA, abandonando de manera definitiva toda soberanía de la política económica y también de la legitimidad política.

Nuestros resultados acerca de los determinantes de la volatilidad macroeconómica brindaron nuevos conocimientos sobre las diferencias entre Brasil y Argentina. La relación positivas entre volatilidad macroeconómica y flujos de capitales extranjeros por una parte y la apertura comercial dentro del Mercosur por otra parte quedaron confirmadas por las estimaciones sobre el conjunto de los países del Mercosur. Un estudio que distingue los dos subgrupos (Brasil-Paraguay y Argentina-Uruguay) hace surgir determinantes diferentes según los grupos de países. La variación de los flujos de capitales extranjeros tiene una relación positiva con la volatilidad macroeconómica en Argentina y en Uruguay, no así en Brasil y Paraguay. Los efectos de la apertura comercial regional y global tienen signos opuestos entre los dos subgrupos igual que los efectos de las políticas de cambio y las IED. Estas diferencias dan cuenta una vez más de la heterogeneidad de los países del Mercosur y las diferentes respuestas de sus economías frente a la globalización y la inte- 
gración regional. Brasil aparece más en condiciones de controlar la volatilidad macroeconómica (menor que en Argentina) por su política monetaria, de cambio, y el rol de las IED en el desarrollo productivo e industrial del país. En cambio, Argentina se halla más sujeta a la vulnerabilidad externa de los flujos de capitales extranjeros y las acciones que puede emprender (política monetaria, financiarización de la economía, régimen de cambio) tienden a acelerar la volatilidad macroeconómica.

El relativo éxito de Brasil en el control de la volatilidad macroeconómica (que condujo al país prácticamente hacia una fase de estagnación desde 1999) no debe confundirse con un éxito en cuanto a las condiciones de trabajo y la vulnerabilidad social, la pobreza y las desigualdades, que son las más importantes del mundo. La latinoamericanización de Argentina (degradación de los indicadores sociales, que estaban al nivel de los países del Centro, a valores cercanos a los de sus vecinos latinoamericanos) fue también uno de los hechos notables de la experiencia de más de diez años de integración regional abierta y financiarizada. Estos aspectos constituyen la segunda serie de resultados de nuestro trabajo.

\section{REgIONALISMO ABIERTO AL SERVICIO DE LA TRANSFORMACIÓN PRODUCTIVA CON EQUIDAD Y VULNERABILIDAD SOCIAL}

El segundo objetivo del Regionalismo Abierto al servicio de la Transformación Productiva con Equidad (TPE) consistía en teoría en asegurar mejores condiciones de trabajo y una mejor protección social gracias al crecimiento sostenido y estable. Las conclusiones de un modelo en economía abierta establecieron la transferencia del riesgo macroeconómico de los flujos de capitales hacia el factor trabajo menos móvil geográficamente (ver Gráficos 4, 5 y 6).

\section{Gráfico 4 - Tasa de desempleo (\% PEA)}

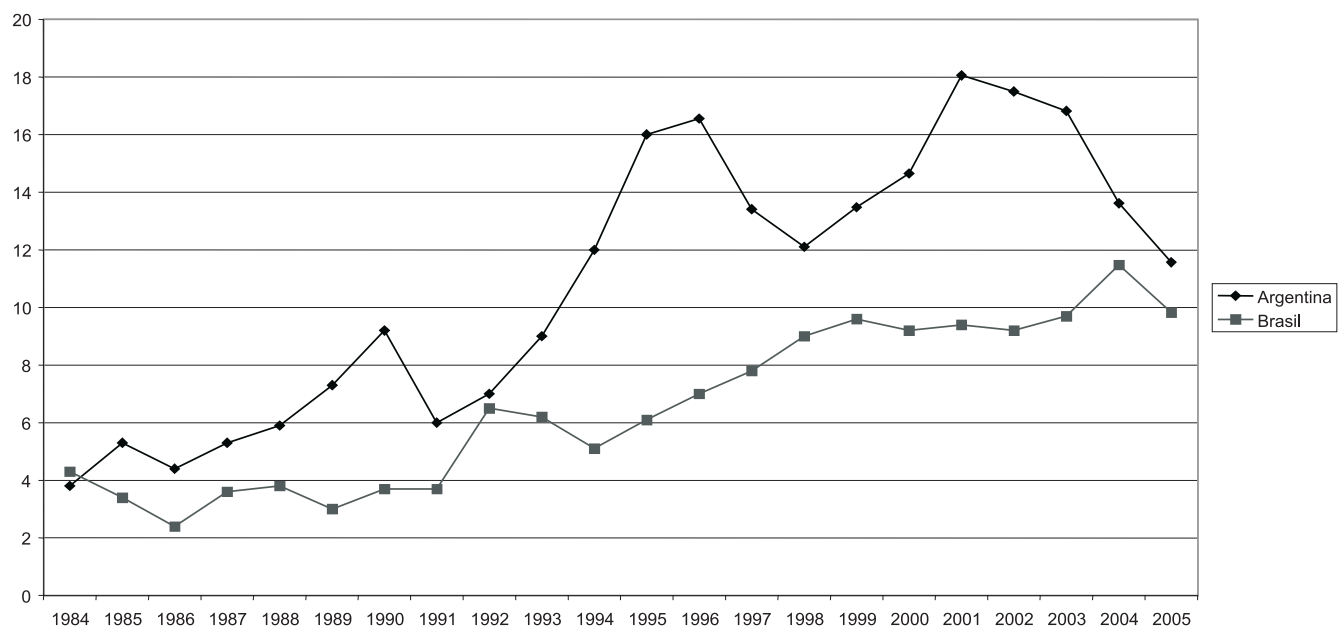

FUENTE: Ecowin, 2006. 


\section{Gráfico 5 - Argentina: Proporción de individuos pobres e indigentes} (\% pop. total) 1991-2002

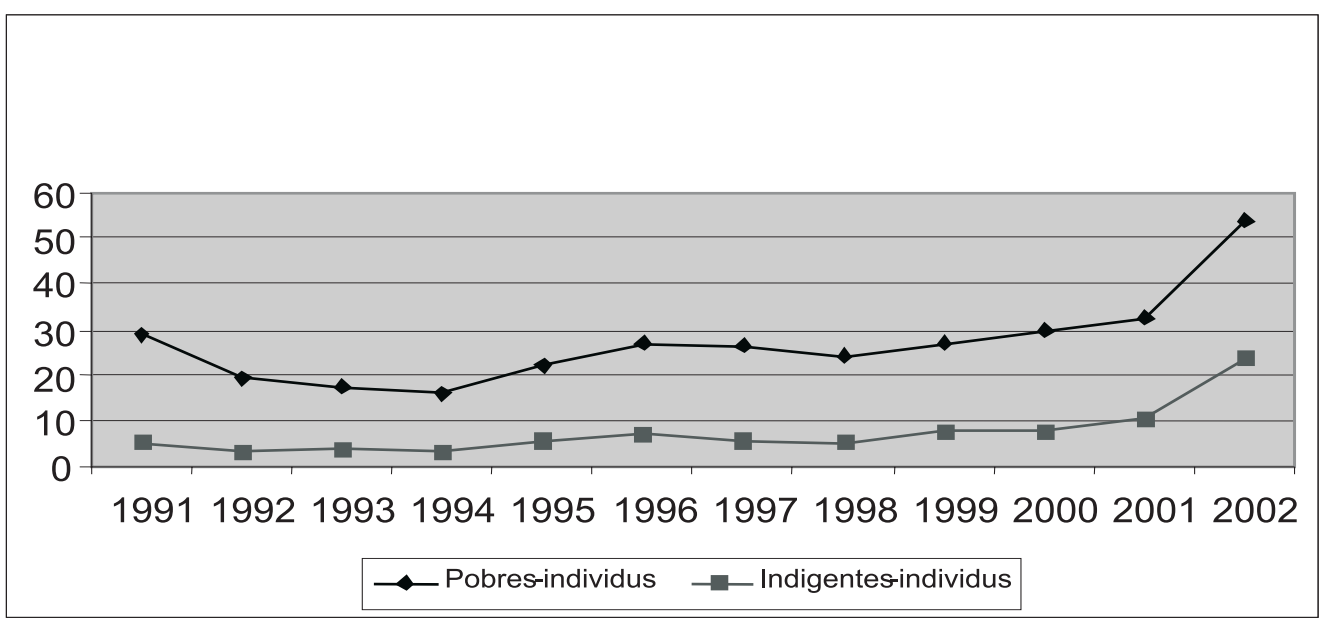

FUENTE: INDEC

\section{Gráfico 6 - Brasil: Proporción de pobres e indigentes} (\% pop. total) 1992-2001

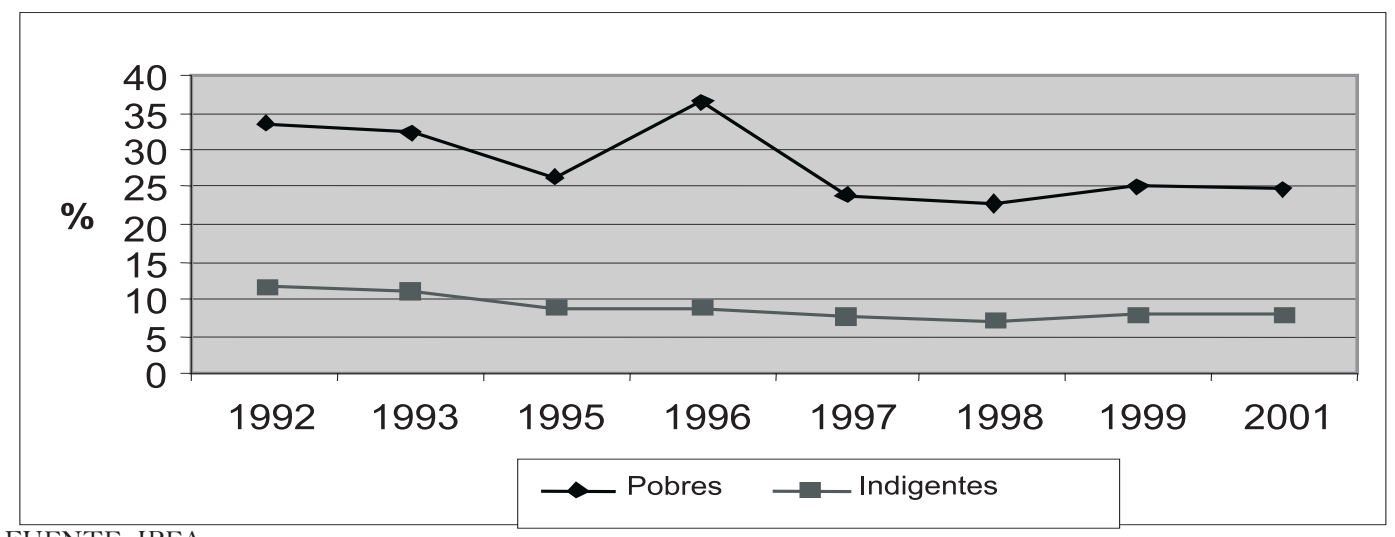

Los efectos positivos de la apertura proclamados por el enfoque liberal encuentran, en el caso del Cono Sudamericano, una información sobre la base empírica. El estudio de la informalidad y de sus factores explicativos mostraron la influencia de los capitales extranjeros y la dependencia, especialmente en Argentina. El carácter financiarizado del Regionalismo Abierto del Mercosur y sus efectos negativos en el mercado de trabajo quedan de esta forma confirmados. El análisis de la descomposición de la tasa de crecimiento de los salarios reales aporta muchos resultados importantes a pesar de ciertos límites debidos a problemas de disponibilidad de datos. Mostramos en particular que el principal factor de la volatilidad de los salarios reales se debía a la diferencia entre las tasas de crecimiento del PBI y de los salarios reales medios. La distribución de los frutos del crecimiento y de la 
productividad está averiada en el Cono Sur, hecho que pone profundamente en cuestión la TPE y la apertura de las economías de la zona. Aún en períodos de crecimiento del PBI y de la productividad del trabajo, estas mejoras no se reflejaron en los salarios reales, poniendo en tela de juicio a las teorías liberales. Las implicancias sobre las políticas macroeconómicas son importantes pero la cuestión de la distribución de los ingresos más igualitaria que responde mejor a un principio de homogeneidad social (preferible al concepto de "equidad") nos conduce a otro debate esencial y vasto. Esencial, porque, como observa B. Lautier, "si bien la estabilización macroeconómica, la reducción de la desigualdad de los ingresos primarios y el desarrollo interno son condiciones necesarias para reducir la pobreza en América Latina, no son condiciones suficientes. Trazan el marco de la emergencia de una verdadera seguridad social pero no dan el contenido". (LAUTIER, 2003, p. 56) El debate es amplio, dado que necesita, para no sostenerse sólo en políticas de lucha contra la pobreza individual, la definición del conjunto de propuestas normativas para una protección social mutualista y universal. Estas propuestas están presentadas en Lautier (2003). Ver también Calcagno y Calcagno (1995, p. 212 y ss) y Tavares y Belluzzo (2002, p. 176). C. Mesalago (1992) trata los mitos y realidades alrededor del importante tema - en América Latina, y también en Europa - de la reforma de las jubilaciones. Las principales ventajas de las reformas liberales via privatización del sistema de jubilaciones son evaluadas a partir de una amplia muestra de países de América Latina durante los años 1990. La casi totalidad de lo que el autor llama mitos (libertad de elección, mejores jubilaciones, efectos positivos sobre el ahorro nacional, etc.) no queda confirmada empíricamente, provocando entonces el debate acerca de este episodio de la política social mutualista y universal.

La ausencia de Trabajo Decente y el incremento de la vulnerabilidad social que pusimos en evidencia en el Cono Sur son factores que permiten un cuestionamiento de las opciones liberales durante mucho tiempo seguidas en estos países. Ni las elecciones de Lula en Brasil o de N. Kirchner en Argentina ni el fracaso del Regionalismo Abierto al servicio de la TPE representan sin embargo una garantía para un cuestionamiento profundo y duradero de la orientación de la política macroeconómica y social en el Merco$\operatorname{sur}^{(2)}$. El tipo de integración regional que se aplicó en el Mercosur le dejó la mejor parte a los países del Centro; los países del Mercosur debieron conformarse con un crecimiento inestable, excluyente, y una gran vulnerabilidad económica, social y política.

Las enseñanzas que nos brindan las fallas y límites del Regionalismo Abierto al servicio de la Transformación Productiva con Equidad definen el contorno de otro tipo de integración regional, sin por ello pretender constituir un catálogo de recetas y de puntos de un programa económico. Se tratará de exponer ciertos elementos que no hemos podido desarrollar en este trabajo pero que constituyen varios ejes de investigación para el futuro. Estos elementos proponen un retorno a ciertas ideas originales del pensamiento estructuralista de la CEPAL acerca de la integración latinoamericana aunque procediendo a las necesarias y numerosas adaptaciones para la nueva configuración de la economía mundial.

(2) Al contrario, al terminarse los mandatos de Lula en Brasil y Kirchner en Argentina, podemos decir que la ruptura que fue declarada por los dos candidatos antes de asumir se pareció mucho más a una politica de continuidad con, es verdad, algunos cambios marginales. 


\section{Para una ReEstructuración Productiva latinoAmericana CON HOMOgeneidad social como alternativa al Alca}

Las enseñanzas acerca del agotamiento de la ISI y el fracaso del Regionalismo Abierto al servicio de la Transformación Productiva con Equidad, abren el camino a varias pistas de investigación para una integración sin desintegración ${ }^{(3)}$. Hay que recortar el debate alrededor del oxímoro que constituye el Regionalismo Abierto, privilegiando claramente el mercado interno regional. No se trata de autarquía pero si de un proteccionismo selectivo permitiendo una diversificación de las economías ${ }^{(4)}$ y un mejor trato al mercado interno regional y a los poblaciones que sufren graves necesidades. También se trata de diversificar los socios comerciales y de no conformarse con las migas que dejan los países del Centro sufriendo los shocks externos (financieros y reales). Estas consideraciones ya fueron mencionadas por R. Prebisch, cuando escribía "la industrialización se desarrolló alrededor de comportamientos coagulados (ISI), el mercado común latinoamericano se impone como otro medio que permite alivianar el estrangulamiento externo y promover la viabilidad de este proceso de industrialización”. (PREBISCH, 1963, p. 107)

Ya mencionamos en el trabajo el interés por desarrollar las relaciones con los otros países del sur, como China, India, Sudáfrica. Hausmann y Gavin (1996) plantearon también interesantes propuestas, como la diversificación de la producción y la constitución de un fondo de estabilización, pero este tipo de medidas se revelan inoperantes a corto plazo y propensas a las crisis. Algunas políticas anticíclicas resultan interesantes (J. A. Ocampo, R. Ffrench-Davis), pero únicamente si se integran en momento de una nueva división internacional del trabajo de América Latina (J. Katz) ya no de padecimiento sino activamente decidida y aplicada a escala regional. Para lograr tal inserción en la economía mundial, la política en materia de tecnología es fundamental y debe estar de entrada definida a partir del objetivo de mejorar la homogeneidad social. En particular, no debe sacrificar ni el mercado interno ni el mercado regional en beneficio de algunos sectores exportadores controlados por un pequeño número de conglomerados nacionales o grandes firmas multinacionales. Nos remitimos en esto al enfoque de A. F. Calcagno y A. E. Calcagno (1995, p. 253):

En un modelo de desarrollo con homogeneidad social, el concepto de competitividad se confunde con el de desarrollo. Las etapas necesarias para alcanzar esta competitividad estructural o sistémica no difieren de aquellas necesarias para un desarrollo sostenido. Las inversiones específicas para alcanzar una capacidad exportadora en ciertos sectores específicos representan poco en comparación con la inversión total que debe realizar un país en el marco de un desarrollo integrado: rutas, educación, salud, alojamiento, industrias de base, investigación, servicios públicos, energía. En definitiva, la competitividad no se limita al nivel de los costos y de la productividad de las empresas sino que depende también del sistema educativo, de la infraestructura tecnológica, de las relaciones entre empleadores y empleados (relación

(3) Ver Salama (1999a).

(4) Ver Salama 2006 y Rodrik, 2005. 
salarial en el enfoque regulacionista) del aparato institucional público-privado, del sistema financiero, etc. En consecuencia, tal desarrollo requiere una política activa y global que sólo el Estado puede llevar a cabo(5).

Las políticas de inserción de los años 1980-1990 privilegiaron el desarrollo "hacia fuera”, abandonando prácticamente el mercado interno y las poblaciones en manos de grandes grupos nacionales concentrados y de firmas multinacionales ${ }^{(6)}$ sin que el Estado intervenga. Del lado de la oferta, las Pequeñas y Medianas Empresas (Py-MES) se vieron gravemente afectadas por la forma de integración regional abierta y financiarizada. Sin embargo, resultan actores claves tanto en los bakckward linkages como en los forward linkages $^{(7)}$. En los países desarrollados, las PyMES conocieron un crecimiento y una consolidación orgánica y selectiva, según la expresión de F. Fajnzylber (1983). El autor estructuralista precisa:

Orgánica, porque las Py-MES se encuentran articuladas como proveedoras de partes y piezas o de productos de alta tecnología a las grandes empresas que estimularon su crecimiento. Selectiva, porque no pueden mantenerse más que aquellas que corresponden a este tipo de articulación, las proveedoras de bienes y servicios finales y aquellas que operan en campos donde no hay ventajas de producción ligadas al tamaño (economías de escala).

A la inversa, en el caso de los países subdesarrollados, el crecimiento de las PyMES fue inorgánico y aleatorio, porque fueron las empresas públicas (antes de las privatizaciones), y sobre todo las privadas, las que se establecieron como empresas de gran tamaño, que transfieren a los inputs importados la mayor parte del efecto estructurante e integrado, papel que las PyMES deberían haber desempeñado". Esta ruptura de las cadenas productivas (o desverticalización) generó una concentración y centralización de la producción y la desaparición de un número considerable de PyMES sin embargo innovadora ${ }^{(8)}$. En el contexto de globalización financiera y de inestabilidad sistémica, podemos interrogarnos acerca de la capacidad de sostener y favorecer a las PyMES. Nuevamente, la ausencia o debilidad de política industrial que busca que las PyMES promuevan una estructura productiva integrada y complementaria da la medida de los esfuerzos que deben realizarse. Es el Estado que debe hacerse cargo de esta promoción de las PyMES, permitiéndoles acceder a un apoyo financiero (política de crédito a las PyMES) y técnica (via centros de conocimiento y de investigación y desarrollo). La situación de penuria en la investigación pública, el cierre o la deslocalización en los países del Centro de las estructuras de Investigación y Desarrollo son razones que hacen de este punto un elemento esencial de la estrategia de competitividad estructural. El activismo en materia tecnológica no puede conformarse con el aporte de las firmas multinacionales implantadas localmente ni con la imitación de

(5) Ver también Fajnzylber (1983) y Calcagno y Calcagno (1995, p. 164-167).

(6) El caso del sector alimenticio en Argentina y en Brasil resulta notable, puesto que el mercado está concentrado en manos de la grandes firmas multinacionales (Danone, Nestlé, Unilever, etc.) y algunos grupos nacionales. Ver Basualdo (1999) y Gonçalves (1999, p. 161 y 172).

(7) Ver Hirschman (1958, p. 100 y ss).

(8) Ver Fajnzylber (1983, p. 411-413), Katz (2001) y Kuwayama y Duran Lima (2003, p. 51-53). 
técnicas para proveer de tecnologías apropiadas a un desarrollo duradero que respete cierta homogeneidad estructural ${ }^{(9)}$ (ver Gráfico 7).

Gráfico 7 - América Latina y el Caribe: índices de innovación y de alerta tecnológica en comparación con otras regiones en desarrollo, 2005

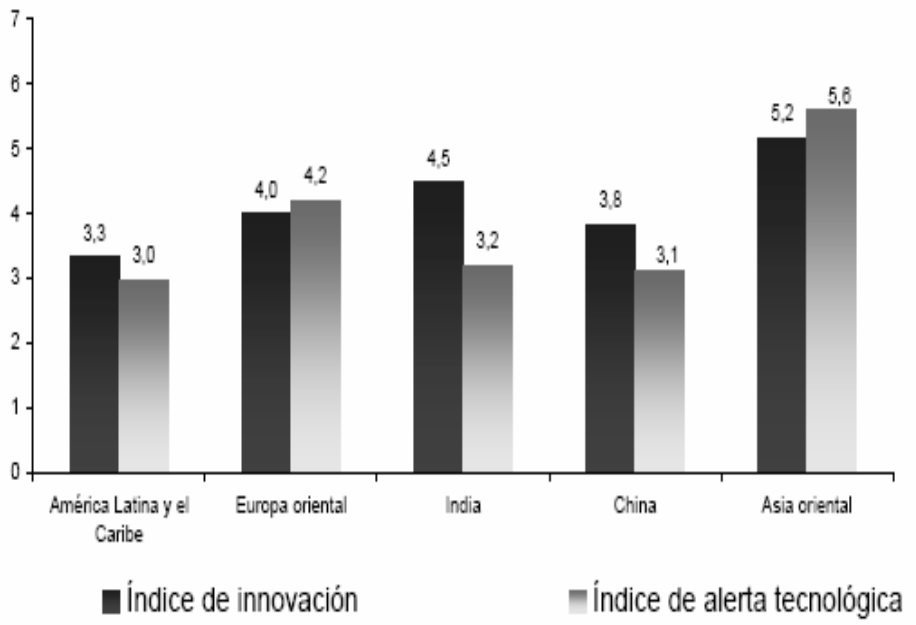

Fuente: Foro Económico Mundial, Global Competitiveness Report 2005-2006, Nueva York, Oxford University Press, 2006.

a Los indices abarcan valores de cero a siete. Los cercanos a cero indican una mayor debilidad, en tanto que valores elevados dan cuenta de una fortaleza.

FUENTE: CEPAL 2006-1, p. 23.

A escala regional, los resultados en términos de ciencia, de tecnología, de innovación y de investigaciones pueden considerarse solamente si una política consecuente se expresa claramente. En los campos de la energía nuclear, de la biotecnología o de la informática, programas de asociación entre Argentina y Brasil resultan necesarios para adquirir una competitividad estructural, en el sentido empleado por F. Fajnzylber. Estas políticas horizontales, sectoriales y regionales de innovación podrían articularse, como propone J. Katz, con las políticas de competitividad gracias a una nueva institución, la "Agencia de Competitividad y de Innovación”. Rompiendo con la lógica del mercado en dicho campo, esta institución tendría como fin facilitar una mayor implicancia local en la creación y difusión

(9) Parece exagerado el optimismo de la CEPAL 2004, cuando pretende que América latina se desarrolle hacia afuera compitiendo frente a los demás paises subdesarrollados cuando el subcontinente muestra señales de innovación y alerta tecnológica tan bajos. 
de nuevos conocimientos, y revitalizar cadenas productivas muy afectadas por la apertura comercial. Según J. Katz (2000, p. 74),

... un nuevo reparto entre público y privado de las acciones tecnológicas e institucionales resulta hoy necesario si los países latinoamericanos queremos asegurarnos de que la transición hacia economías más abiertas al mundo y más desreguladas sea compatible con un mejor ritmo de creación y de difusión de nuevos conocimientos tecnológicos, de ganancias de productividad más importantes, y una mayor equidad y democratización de nuestras sociedades.

Este tipo de inserción en la economía mundial que apunta a la homogeneidad social a escala latinoamericana resalta el rol de las instituciones tanto políticas como monetarias. Como lo señaló C. Furtado (1968, p. 282-283):

... la integración económica constituye un esfuerzo que busca maximizar las economías de escala de producción en función de la técnica disponible, buscando estimular las economías de aglomeración o compensando correctamente los efectos negativos que podrían producirse sobre colectividades específicas (...) Así, la teoría de la integración constituye una etapa superior de la teoría del desarrollo y la política de la integración adquiere la forma de una política de desarrollo. La planificación de la integración será, en consecuencia, la forma más compleja de coordinación de las decisiones económicas. En los que concierne a los países subdesarrollados, la integración no planificada conlleva necesariamente una agravación de los desequilibrios regionales y una mayor concentración geográfica de los ingresos.

La importancia de coordinación macroeconómica remite por supuesto al rol del Estado como institución reguladora de los mercados y garante de la homogeneidad social. Los estructuralistas latinoamericanos ya habían percibido esta idea. Así, según R. Prebisch (1963, p. 107),

... sería erróneo considerar que los instrumentos que consagra el Tratado del Mercado Común (latinoamericano) no son eficaces en sí mismo. En efecto, lo son, pero necesitan de importantes decisiones políticas para que esta eficacia se traduzca en hechos que resistan la estanflación que reina [fin de la ISI].

Las investigaciones acerca de la creación de una moneda única en el Mercosur van también en el sentido de una afirmación de soberanía monetaria por mucho tiempo dependiente de la moneda estadounidense (especialmente Argentina). Los partidarios de la dolarización de las economías latinoamericanas están siempre activos, pero su opinión significaría pura y simplemente el abandono de la soberanía nacional para ventajas inciertas y en beneficio de un grupo muy reducido de personas. Sin embargo, como el caso de la Unión Europea muestra, instituciones políticas regionales fuertes y una moneda soberana no garantizan una homogeneidad social y estructural. La ausencia de política social europea, el nivel del desempleo y la nivelación hacia abajo de los sistemas de protección social en Europa lo certifican. Las observaciones para una reestructuración productiva latinoamericana con homogeneidad social definen varios puntos a favor de un modelo de desarrollo alternativo al ALCA. 


\section{Conclusiones}

Los elementos de políticas de la CEPAL de los años 1950 tenian que ver con un escenario geopolítico, económico y social bien diferente. La incorporación de Venezuela en diciembre del 2005 y de manera formal en 2006 modifica el equilibrio (o desequilibrio) entre las economías latinoamericanas. El tema energético, con el elevadisimo nivel de precios de los hidrocarburos, hace que Venezuela esté con enorme liquidez.

Del lado Venezolano, la voluntad de fortalecer la integración latinoamericana es muy clara pero tambien ese país busca respaldo político en caso de un eventual enfrentamiento (directo o indirecto) con los EEUU. De la misma manera, el acercamiento de Bolivia y sus reservas de gas con el Mercosur ampliado cambia el escenario sin quitar dudas e incertidumbre como con el caso de la empresa Petrobras en este país (25\% del PIB) y los acuerdos pre-elección (de 2006) entre los presidentes Morales y Lula.

Al nivel del subcontinente, el fuerte rechazo al ALCA a través de la cumbre de Mar del Plata en diciembre del 2005, dejó más espacio por lo menos para pensar en otra integración latinoamericana. Sin embargo, este espacio mayor no significó mayor esfuerzo común para suavizar las tensiones entre países de la zona como lo muestran las peleas diplomáticas y comerciales de estos últios años:

- entre Argentina y Brasil acerca del respaldo para la sede permanente en la ONU que pedia Brasil a Argentina o al apoyo pedido a Brasil por Argentina en sus negociaciones tensas con el FMI para el canje de parte de su deuda;

— entre Brasil y Uruguay sobre el apoyo al candidato uruguayo al puesto de presidente de la $\mathrm{OMC}$;

- entre Argentina y Uruguay acerca de las papeleras. Es significativo que en ese caso se haya desarrollado una queja en la OMC y no de manera interna en el Mercosur. Esto es un indicador claro de que el mecanismo de solución de controversia no funcionó. Tampoco es soprendente que en esta situación tensa, los esfuerzos de EEUU para firmar acuerdos bilaterales con varios paises de la región sea considerado seriamente, especialmente por Uruguay, miembro fundador del Mercosur.

Los superavits fiscales y el nivel elevado de las reservas de los grandes paises del subcontinente son una oportunidad en periodo de ausencia de crisis económica abierta para redefinir el rumbo de la inserción internacional de la región, del tratamiento a dar a la inversión en infraestructura, en el mercado interno que sufre de tantas necesidades. El escenario mundial es bastante favorable (precio materias primas elevadas, fuerte demanda de China, etc.) pero el caracter duradaero es muy incierto. La oportunidad de crear un Banco del Sur (apoyado por Venezuela, Argentina) y/o un Fondo Monetario del Sur esta siendo discutida pero los primeros elementos muestran que se trata más de un substituto regional al Banco Mundial o al BID que de un Banco o Fondo alternativo ${ }^{(10)}$.

(10) Ver los documentos del seminario "Sobre deuda pública, auditoria popular y alternativas de ahorro e inversion para los pueblos de América latina”, Caracas, Venezuela, 22, 23 y 24 de septiembre de 2006. <http://www.oid-ido.org/oidido.php3?id_article $=371>$. 
El tema de la deuda que tuvo tanto peso en los 1980s (pero también en los 1990 y hasta el inicio del tercer milenio con la crisis abierta en Argentina y el default de 2001 a 2005) sigue siendo un tema clave que puede servir de indicador entre dos políticas alternativas: continuidad o ruptura con el modelo néoliberal. Los pagos anticipados al FMI por parte de Brasil y Argentina en 2005 (respectivamente 15,5 y 9,8 miles de milliones de USD) muestran claramente la opción de continuidad y de busqueda de la aprobación de los mercados e Instituciones financieras internacionales que se esta siguiendo. La supuesta busqueda de mayor autonomia pasa para los gobiernos actuales (Lula y Kirchner) por el pago de una deuda que tuvo como origen las dictaduras militares y actos fraudulosas en ciertos $\operatorname{casos}^{(11)}$.

No podemos terminar este analisis sin mencionar — aunque séa de manera rapida la propuesta del ALBA (Alternativa Bolivariana para las Américas) ${ }^{(12)}$. La salida del sistema capitalista financiero y comercial mundial puede ser una vía interesante a considerar cuando vemos algunas consecuencias de este sistema en terminos de pobreza, desigualdades, violencia. Sin embargo, los ejemplos de intercambio entre Venezuela y Cuba (petroleo contra servicios médicos y de educación) o entre Bolivia y Cuba (apoyo técnico) no pueden ser solo acciones puntuales posibles por el alto precio del petroleo que por cierto entra en el comercio mundial (gracias esencialmente a las compras de los EEUU a Venezuela). ¿Que pasará cuando el precio del petroleo disminuya? Estas acciones puntuales limitadas al sector social tendrian que abarcar la esfera productiva con políticas públicas que permitan la redefinición al nivel directamente regional (al contrario de lo que se hizo durante la Industrialización por Sustitución de Importaciónes) de la división internacional del trabajo desde mucho tiempo (o tal vez desde siempre?) a favor de las economías del Norte y de sus grandes empresas transnacionales ${ }^{(13)}$. Sin volver a la fórmula de los estructuralistas de la CEPAL de los 1950 s de "desarrollo hacia adentro", se trata de no considerar los 500 milliones de latinoamericanos apenas como una demanda insolvente, sino como las fuerzas vivas que podran construir un mundo menos desigual y más próspero para todos (y todas) en América Latina.

\section{REFERENCIAS BIBLIOGRAFICAS}

BIELSCHOWSKY, Ricardo. Cincuenta años del pensamiento de la CEPAL: una Reseña. CEPAL 1998b, ouvrage cité, 1998.

CALCAGNO, Alfredo. El universo neoliberal. Buenos Aires: Allianza, 1995.

CEPAL. Panorama de la inserción internacional de América Latina y el Caribe. Santiago du Chili, sep. 2006-1.

Estudio económico de América Latina y el Caribe. Santiago du Chili, sept. 2006-2.

Balance preliminar de las economías de América Latina y el Caribe. Santiago du Chili, déc. 2005-1.

\footnotetext{
(11) Ver el Caso Olmos en Argentina o los trabajos del CADTM y del OID sobre la deuda illegitima. <www.cadtm.org y www.oid-ido.org>.

(12) Sobre ese tema, ver los articulos en la pagina: <http://risal.collectifs.net/mot.php3?id_mot $=458>$

(13) Las grandes empresas y conglomerados nacionales de los paises del Sur también tuvieron interes en mantener esta situación de dependancia.
} 
du Chili, 2005-2.

Objetivos de dessarrollo del milenio. Una mirada desde América Latina y el Caribe, Santiago Desarrollo productivo en economías abiertas. Santiago du Chili, jui. 2004.

. Une décennie d'ombres et de lumières. L'Amérique Latine et les Caraïbes dans les années 90. Santiago du Chili, aoû. 2003.

Equidad, desarrollo y ciudadanía. Versión définitiva. Santiago du Chili, 2001.

Cincuenta años de pensamiento en la CEPAL. Santiago du Chili, Textos seleccionados, 2 tomes, FCE - CEPAL, 1998.

El regionalismo abierto en America Latina y el Caribe; la integración economica al servicio de la Transformación Productiva con Equidad. Libros de la CEPAL, Santiago du Chili, n. 39, 1994.

Equidad y transformación productiva: un enfoque integrado. Libros de la CEPAL, Santiago du Chili, 1992. 1990.

Transformación productiva con equidad. Libros de la CEPAL, Santiago du Chili, n. 25,

FAJNZYLBER, Fernando. Industrialización en América Latina: de la caja negra al casillero vacio. Cuadernos de la CEPAL, n. 60, 1990 et repris dans CEPAL, 1998, ouvrage cité. Ecowin, Base de dados, 2006.

\section{La industrialización trunca de América Latina. Mexico: Nueva Imagen, 1983.}

Oligopolio, empresas transnacionales y estilos de desarrollo. In: FAJNZYLBER, F. (coord.). Industrialización e internacionalización en Américas Latina. Mexico, Ed. FCE - Trimestre Económico, 1980.

FURTADO, Celso. Desarrollo y estancamiento en América Latina: un enfoque estructuralista. 1966, repris dans Prebisch, R. et al 1969, ouvrage cité.

GIRALDO, Cesar. Protección social y poder financiero. Thèse de doctorat, miméo, Université Paris 13, 2006.

GUILLEN, Romo. De la pensée de le CEPAL au néo-libéralisme, du néo-libéralisme au néo-structuralisme, une revue de la littérature sud-américaine. Revue Tiers Monde, t. XXXV, n. 140, PUF, 1994.

HIRSCHMAN, Albert O. Grandeur et décadence de l'économie du développement. (1981). In: HIRSCHMAN A. O. L'économie comme science morale et politique. Paris: Col. Hautes Études, Gallimard-Le Seuil, 1984 .

HOUNIE, Adela; PITTALUGA, Lucía G.; SCATOLI, Fabio; PORCILE, Gabriel. La CEPAL y las nuevas téorias del crecimiento. Revista de la CEPAL, Santiago du Chili, n. 68, ago. 1999.

KURI, Gaytán. Technogical change and structuralist analysis. CEPAL Revierw, Santiago du Chili, n. 55, avril. 1995.

MUSACCHIO, Andres. La crisis del MERCOSUR y las alternativas de salida. Presentado en el Seminario Internacional: Integración y desarrollo sustentable. La nueva geografía de los recursos, la economía y el poder. CLAES - D3E. Montevideo, 14-15 julio. 2005.

Argentina ante el Alca: solución a los dilemas del crecimiento o agravamiento de los problemas? Realidad Economica, v. 182, n. 1, p. 16-33, 2001.

140 
PINTO, Anibal. Notas sobre los estilos de desarrollo en América Latina. Revista de la CEPAL, n. 1, 1976, et repris dans CEPAL 1998, ouvrage cité.

La concentración del progreso técnico y de sus frutos en el desarrollo latinoaméricano. 1965, In: PREBISCH, Raúl et al. América Latina, ensayos de interpretación económica. Santiago du Chili: Universitaria, 1969.

PREBISCH, Raul et al. América Latina, ensayos de interpretación económica. Santiago du Chili: Universitaria, 1969.

RODRIK, Dani. Políticas de diversificación económica. Revista de la CEPAL, Santiago du Chili, n. 87, déc. 2005.

SALAMA, Pierre. Le défi des inégalités. Amérique Latine/Asie, une comparaison économique. Paris: La Découverte, 2006.

Riqueza y pobreza en América Latina; la fragilidad de las nuevas políticas económicas. Mexico: FCE — Universidad de Guadalajara, 1999.

SALAMA, Pierre; MARQUES-PEREIRA, Jaime; LAUTIER, Bruno. Régime de croissance, vulnérabilité financière et protection sociale en Amérique Latine. Serie Financiamento del Desarrollo de la CEPAL, n. 140, 2003.

SALUDJIAN, Alexis. Le Régime d'Accumulation dans le Cône Sud-Americain depuis les années 1990. Crise de régulation ou crise d'accumulation? Revue de la Régulation: Capitalisme, Institutions Pouvoirs, n. 1, p. 1-23. <http://regulation.revues.org/document1313.html. 2007-1>.

. Las elecciones francesas de 2007: ¿qué políticas económicas para qué política?, El Debate Político. Revista Iberoamericana de Análisis Político, Buenos-Aires, Fondo de Cultura Económica, n. 6/7, Año 4, p. 213-225, 2007-2.

Pour une autre intégration sud-américaine: critiques du Mercosur néo-libéral. Paris: Editions L’Harmattan, Collection Recherches Amérique Latine, 2006-1.

. Flux de capital et intégration régionale: une étude econométrique des principaux déterminants de l'instabilité macro-économique dans le cône sud-américain. Problemas del Desarrollo, México, n. 147, p. 107-132, oct./dec. 2006-2.

. Del Mercosur al ALCA: críticas al modelo liberal de nuevo regionalismo de la BID. Problemas del desarrollo. Revista Latinoamericana de Economía, UNAM-IIE, México, n. 141, p. 9-25, avr./jui. 2005-1.

. Hacia otra integración sudamericana: críticas al Mercosur neoliberal. Editions Libros del Zorzal, Colección Ensayos, Buenos-Aires, Argentina, thèse publiée, remaniée et traduite en espagnol, 352 p. 2005-2.

. Le modèle de la croissance excluante et l'insécurité économique dans le Mercosur depuis 1990. Revue Tiers Monde, Paris, n. 184, PUF, p. 883-905, oct./déc. 2005-3.

. Trajectoires de Croissance et Volatilité Macro-économique dans le Mercosur: quelques eléments d'analyse empirique. Revue Tiers - Monde, n. 179, Paris, PUF, p. 595-615, jui./sep. 2004.

STIGLITZ, Joseph. Le cap des réformes. Vers un nouveau programme pour l'Amérique Latine. Revista de la CEPAL, Santiago de Chile, Numero Especial (écrit en 2002), 2005.

. La grande désillusion. Paris: Fayard, 2002. 
Alexis Saludjian — Cadernos PROLAM/USP ( Ano 7 - Vol. 2 - 2008) p. 127- 142

SUNKEL, Oswald. Development from within: toward a neostructuralist approach for Latin America. Londres, New York: Lynne Riener, Boulder, 1993.

SUNKEL, Owald; ZULETA, G. Neoestructuralismo versus neoliberalismo en los años 1990. Revista de la CEPAL, Santiago du Chili, n. 42, p. 35-53, 1990. 